\title{
Incidence of intravascular insertion in thoracic epidural catheterization by using real time fluoroscopy
}

\author{
Sae Young Kim, Yun Young Kim, and Ae Ra Kim \\ Department of Anesthesiology and Pain Medicine, School of Medicine, Keimyung University, Daegu, Korea
}

Background: Epidural analgesia is commonly used to provide several types of pain relief. Although this technique has been widely used with many advantages, currently the complications appear to be increasing. Especially, inadvertent intravascular cannulation and intravascular local anesthetic administration can lead to fatal consequences.

Methods: Data was collected on 296 patients undergoing elective thoracic or abdominal surgery. Two detection methods were utilized to confirm the epidural intravascular cannulation; flashback and aspiration of indwelling catheter, and injection of a contrast agent through the catheter under fluoroscopy were used to guide the placement of the catheter and to examine the intravascular cannulation.

Results: Epidural intravascular cannulation was reported in 4 out of 296 cases (1.4\%), and 1 patient underwent subdural cannulation. Among the 4 cases of epidural intravascular cannulation, two were confirmed by the flashback and aspiration methods, while the remaining cases were only detected by real time fluoroscopy.

Conclusions: In this study, inadvertent epidural intravascular cannulation occurred by as much as $1.4 \%$ of thoracic epidural catheterization. Utilizing real time fluoroscopy in addition to flashback and aspiration can enhance the sensitivity of detection. (Korean J Anesthesiol 2012; 62: 251-255)

Key Words: Analgesia, Blood vessels, Catheters, Epidural space, Thorax.

\section{Introduction}

Epidural analgesia is commonly used to control low back pain, delivery pain, postherpetic neuralgia, cancer pain, and intraoperative and postoperative pain. It involves catheter installation to continuously inject drugs [1-3]. Compared to oral or intravenous drug administration, the use of epidural analgesia for the purpose of intraoperative and postoperative pain control has been gradually increasing due to its advantages such as increased cardiopulmonary functions, decreased dose of analgesic agents during surgery, fast recovery of gastrointestinal functions after surgery, fast tracheal extubation, and improved motility. However, its frequency of complications also increases in proportion to its increased utilization. Complications of epidural analgesia include infection, bleeding, intravascular administration, nerve injury, and disc injury [4].

Received: May 16, 2011. Revised: 1st, August 15, 2011; 2nd, October 10, 2011; 3rd, October 14, 2011. Accepted: October 17, 2011.

Corresponding author: Sae Young Kim, M.D., Department of Anesthesiology and Pain Medicine, School of Medicine, Keimyung University, 194, Dongsan-dong, Jung-gu, Daegu 700-712, Korea. Tel: 82-53-250-7287, Fax: 82-53-250-7240, E-mail: mandell@naver.com

(c) This is an open-access article distributed under the terms of the Creative Commons Attribution Non-Commercial License (http:// creativecommons.org/licenses/by-nc/3.0/), which permits unrestricted non-commercial use, distribution, and reproduction in any medium, provided the original work is properly cited. 
Among such complications, unintended epidural intravascular cannulation causes not only the failure of epidural analgesia, but also serious complications such as systemic toxicity by a local anesthetic agent, convulsion, heart attack, and death due to intravascular drug injection [5].

In catheterization for a continuous epidural block, the incidence rate of intravascular cannulation in the lumbar level was reported to be $9-10 \%$ [6-9]. For transforaminal epidural injection, the intravascular flow rate was reported to be $20.6 \%$, $8.2 \%$ and $6.1 \%$ in the cervical, thoracic, and lumbar levels, respectively [10]. However, no study has been conducted to investigate the incidence rate of epidural intravascular cannulation in the cases of cervical or thoracic catheterization. Accordingly, this study was conducted to investigate the incidence rate of epidural intravascular cannulation using realtime fluoroscopy in thoracic epidural catheterization.

\section{Materials and Methods}

This study was conducted on patients who were categorized as American Society of Anesthesiologists physical status I-III and who had undergone thoracic epidural catheterization to control intraoperative and postoperative pain, among those who had undergone thoracotomy or abdominal subcostal incision from September 2009 to April 2011. The patients were given an adequate explanation of the purpose of this study and the study procedures before they signed the informed consent form. Of the total of 310 patients, thoracic epidural catheterization was conducted on 296 patients, except 7 patients who refused to undergo the treatment, 5 patients who showed a laboratory finding of bleeding, and 2 patients who had a previous history

Table 1. Demographic Data of the Study Patients $(\mathrm{N}=296)$

\begin{tabular}{cc}
\hline Characteristics & Values \\
\hline Age (yr) & $60.7 \pm 12.1$ \\
Sex (M/F) & $193 / 103$ \\
Height $(\mathrm{cm})$ & $162.2 \pm 8.1$ \\
Weight $(\mathrm{kg})$ & $60.3 \pm 10.6$
\end{tabular}

The values are shown as mean \pm SD or number of patients. of allergic reaction to contrast agents. The demographic data of the patients are presented in Table 1.

Thoracic epidural catheterization was conducted on the patients in a prone position. The insertion site of the catheter was aseptically disinfected with povidone, and injected with $2 \%$ lidocaine, followed by a one-minute waiting period. Then, an $18 \mathrm{G}$ Touhy needle was inserted into the targeted $6^{\text {th }}$ and $8^{\text {th }}$ thoracic vertebra via the paramedian approach using fluoroscopy (OEC series 9800, General Electronics, USA). The epidural space was identified via the loss of resistance technique using a saline solution, followed by epidural catheter installation. Once the catheter was installed, the potential occurrence of flashback through the catheter was examined for one minute, and then aspiration was conducted to examine the presence of blood. Then, a $3 \mathrm{ml}$ of contrast agent (Iopamino ${ }^{\circledR}$ 370 , Ilsung, Korea) was injected at a rate of $2-3 \mathrm{ml} / \mathrm{sec}$ under real-time fluoroscopy while observing the intravascular cannulation.

\section{Results}

Epidural intravascular cannulation was reported in 4 patients (1.4\%) and subdural cannulation was reported in one patient out of the 296 patients. Among the four cases of epidural intravascular cannulation (Table 2), two cases, which were confirmed by the flashback and aspiration methods, showed that the contrast was only intravascularly shown under a realtime fluoroscopy, and the remaining two cases, which were not confirmed by the flashback and aspiration methods, showed that the contrast spread to both small vessels and the epidural space under real-time fluoroscopy. Re-catheterization was conducted on the four patients who were confirmed to have intravascular cannulation. After confirming that intravascular cannulation did not occur via real-time fluoroscopy, the catheter was fixed.

\section{Discussion}

In this study, thoracic epidural catheterization was conducted using the flashback and aspiration methods via the

Table 2. Data of Patients of Intravascular Insertion in Thoracic Epidural Catheterization

\begin{tabular}{|c|c|c|c|c|c|c|c|c|c|}
\hline & \multirow{2}{*}{$\begin{array}{l}\text { Age } \\
\text { (yr) }\end{array}$} & \multirow{2}{*}{$\begin{array}{c}\text { Sex } \\
(\mathrm{M} / \mathrm{F})\end{array}$} & \multirow{2}{*}{$\begin{array}{c}\text { Height } \\
(\mathrm{cm})\end{array}$} & \multirow{2}{*}{$\begin{array}{c}\text { Weight } \\
\text { (kg) }\end{array}$} & \multicolumn{2}{|c|}{ Location of catheter's tip } & \multicolumn{3}{|c|}{ Methods of detection } \\
\hline & & & & & $\mathrm{R} / \mathrm{L}$ & Spine level & Flashback & Aspiration & Real-time fluoroscopy \\
\hline 1 & 63 & $\mathrm{~F}$ & 156 & 58 & $\mathrm{R}$ & T6 & - & - & + \\
\hline 2 & 23 & M & 171 & 74 & $\mathrm{R}$ & $\mathrm{T} 8$ & + & + & + \\
\hline 3 & 32 & M & 180 & 84 & $\mathrm{~L}$ & T6 & - & - & + \\
\hline 4 & 76 & $\mathrm{~F}$ & 152 & 56 & $\mathrm{~L}$ & $\mathrm{~T} 8$ & + & + & + \\
\hline
\end{tabular}

R: right, L: left, T6: 6th thoracic vertebra, T8: 8th thoracic vertebra. 
catheter and contrast injection under real-time fluoroscopy. The incidence rate of epidural intravascular cannulation in the thoracic level was $1.4 \%$, which was lower than the incidence rate of intravascular cannulation in the lumbar level of $10 \%$ that occurred upon the first epidural block.

When the artery diameter was compared according to the spinal cord level with a risk of intravascular cannulation, the diameter of the artery of the thoracic level was $0.4-0.6 \mathrm{~mm}$, which was smaller than the diameter of the artery of the lumbar level of $0.55-1.2 \mathrm{~mm}$, and was even smaller than the epidural catheter diameter of $0.8-1.2 \mathrm{~mm}$. Thus, the incidence rate of intravascular cannulation was likely to be lower in the thoracic level than in the lumbar level [11]. However, in the epidural block, intravascular contrast injection is conducted via the vein in most cases. The vertebral venous plexus does not have a valve, and is distributed along the entire pathway of the spine. The posteromedial venous plexus of the vertebral venous plexus is present between the posterior epidural and the epidural lipid layer. The posteromedial venous plexus is connected from the neural foramen to the radicular vein where the radicular artery accompanies the spinal nerve [12]. The vertebral venous plexus is dilated for local or systemic reasons, leading to an increase in intravascular injection. In particular, in the cases of increased abdominal pressure such as abdominal tumor or pregnancy, inferior vena cava flow is inhibited and the epidural vein is vasodilated. In addition, the anterior and posterior diameter of the epidural space gradually increases from $3-5 \mathrm{~mm}$ in the thoracic level to 5-6 $\mathrm{mm}$ in the lumbar level [13]. Therefore the lateral area of the epidural space is freely connected to the paraspinal space via the neural foramen, or intra-abdominal pressure is directly delivered via the open neural foramen. Thus, compared to the thoracic level, it is likely that the lumbar level is more affected by the elevation of intra-abdominal pressure, and has an increased possibility of vasodilatation caused by the compression of vein flow in the epidural space, and an increased risk of epidural intravascular cannulation. Local compression and distortion of the epidural lipid may occur due to degenerative changes caused by increased age [14]. These degenerative changes compress venous flow and the vasodilate venous plexus. Although the degenerative changes of the spine increase in proportion to age, intravascular cannulation was observed in various age brackets in this study. It has been reported that the general distribution of the spinal anteromedial venous plexus was homogenous, whereas that of the posteromedial venous plexus, where catheterization is conducted upon the epidural block, varied individually [12]. This result could demonstrate that intravascular catheterization in an epidural block is disproportional to the degenerative changes according to increased age. A further study on the correlation of the epidural venous plexus of each spinal region, particularly the anatomical features of the posteromedial venous plexus with epidural intravascular cannulation would be required to obtain more supportive results.

In this study, the incidence rate of epidural intravascular cannulation in the thoracic level was $1.4 \%$, which was lower than the incidence rate of the intravascular cannulation of $9-10 \%$ that occurred in the lumbar level. No anatomical or radiologic study has been conducted to date on the venous plexus diameter present in the posterior epidural space in which epidural cannulation is conducted. Therefore, a further study is required to investigate this difference in the frequencies of intravascular cannulation.

Many studies have been conducted to examine how to prevent the risk of epidural intravascular cannulation during epidural cather placement. It has been reported that spinalepidural combined analgesia reduces the incidence rate of epidural intravascular cannulation [15]. It has been estimated that the risk of intravascular cannulation could be reduced by inserting the epidural catheter to the center because the lateral area of the epidural space has abundant vascularity. However, it eventuated that no significant difference was observed when comparing the use of the loss of resistance to saline technique and the air technique or when comparing the use of the paramedian approach to the midline approach [16]. It has been reported that saline solution injection prior to catheterization reduced the incidence rate of intravascular cannulation [17], but this is still controversial. In addition, it has been reported that catheterization with a soft and elastic catheter reduced the incidence rate of intravascular cannulation [18].

Various methods have been used to monitor epidural intravascular cannulation, of which direct methods such as aspiration and indirect methods such as injection of adrenalin or fentanyl are typical $[6,7]$. However, the aforementioned methods cannot completely exclude epidural intravascular cannulation when they are used alone [19]. In the case of a single epidural block, it has been recommended that the accurate location of the needle should be identified via real-time fluoroscopy to prevent intravascular cannulation [20,21]. Accordingly, in this study, intravascular cannulation was monitored using realtime fluoroscopy. As a result, intravascular cannulation was shown in four patients out of the 296 patients. Among the four cases of epidural intravascular cannulation, two cases were confirmed by the flashback and aspiration methods. Kim and Shim [22] reported that the detection failure of the blood via the flashback and aspiration methods was attributable to the insufficient vein pressure causing the insufficient blood flow into the catheter contact region and that the blood flow was not observed due to vascular collapse caused by excessive negative pressure particularly in the case of aspiration. Thus, as shown in the results of previous studies, the flashback and aspiration 
methods are unlikely to predict the actual status of the blood vessels.

Intravascular flow can be monitored using Real-time fluoroscopy. However, it is not easy to distinguish the vein from the artery. In general, an intravascular flow with a fast flow rate and moving from the neural foramen to the spinal cord is considered the artery, whereas intravascular flow with a slow flow rate and moving from the neural foramen to the peripheral is considered the vein. However, in actual clinical practices, the two aforementioned intravascular flows are mixed in some cases, and the contrast spreads to the small blood vessels that are barely detected with the eyes toward the spine while moving to the peripheral. Thus, the accuracy of the real-time monitoring of the artery and vein is low [23].

Thoracic epidural catheterization is mainly conducted as a blind technique without the assistance of fluoroscopy. Real-time fluoroscopy after epidural catheterization is not generally conducted in all the cases. In this study, two cases of intravascular cannulation, which were not confirmed by other methods, were confirmed via real-time fluoroscopy.

Digital subtraction imaging was not conducted in this study. This technique is a radiological technique that can clearly visualize the blood vessels surrounded by the osteoid or dense soft tissues. Vascular imaging can be produced using a contrast medium by subtracting a pre-contrast image. Therefore, it is useful in the diagnosis and treatment of vascular diseases. In particular, the monitoring rate of intravascular drug injection is 2-fold higher than other techniques. However, its disadvantages include high cost and being more influenced by the conductor's performance $[24,25]$. As digital subtraction imaging is not generally used due to its high cost, it was not conducted in this study.

In conclusion, in this study, the incidence rate of epidural intravascular cannulation was lower in the thoracic level than in the lumbar level. However, in some cases, epidural intravascular cannulation was not detected by the flashback or aspiration methods. Thus, the utilization of real time fluoroscopy or digital subtraction imaging in addition to flashback and aspiration is recommended to identify intravascular cannulation.

\section{References}

1. Grass JA. The role of epidural anesthesia and analgesia in postpoerative outcome. Anesthesiol Clin North America 2000; 18: 40728.

2. Park WY, Thompson JS, Lee KK. Effect of epidural anesthesia and analgesia on perioperative outcome: a randomized, controlled Veterans Affairs cooperative study. Ann Surg 2001; 234: 560-9.

3. Vad VB, Bhat AL, Lutz GE, Cammisa F. Transforaminal epidural steroid injections in lumbosacral radiculopathy: a prospective randomized study. Spine (Phila Pa 1976) 2002; 27: 11-6.

4. Waldman SD. Management of acute and postoperative pain. In: Pain management. Edited by Waldman SD: Philadelpia, Saunders. 2007, pp 225-39.

5. Tiso RL, Cutler T, Catania JA, Whalen K. Adverse central nervous system sequelae after selective transforaminal block: the role of corticosteroids. Spine J 2004; 4: 468-74.

6. Verniquet AJ. Vessel puncture with epidural catheters. Experience in obstetric patients. Anaesthesia 1980; 35: 660-2.

7. Kenepp NB, Gutsche BB. Inadvertent intravascular injections during lumbar epidural anesthesia. Anesthesiology 1981; 54: 172-3.

8. Paech MJ, Godkin R, Webster S. Complications of obstetric epidural analgesia and anaesthesia: a prospective analysis of 10,995 cases. Int J Obstet Anesth 1998; 7: 5-11.

9. Norris MC, Fogel ST, Dalman H, Borrenpohl S, Hoppe W, Riley A. Labor epidural analgesia without an intravascular "test dose". Anesthesiology 1998; 88: 1495-501.

10. Nahm FS, Lee CJ, Lee SH, Kim TH, Sim WS, Cho HS, et al. Risk of intravascular injection in transforaminal epidural injections. Anaesthesia 2010; 65: 917-21.

11. John MM. Image-guided spine interventions. 2nd ed. New York, Springer. 2010, pp 15-27.

12. Groen RJ, Groenewegen HJ, van Alphen HA, Hoogland PV. Morphology of the human internal vertebral venous plexus: a cadaver study after intravenous Araldite CY 221 injection. Anat Rec 1997; 249: 28594 .

13. James PR. Atlas of image-guided intervention in regional anesthesia and pain medicine. Philadelphia, Lippincott Williams \& Wilkins. 2006, pp 31-52.

14. Hogan QH. Epidural anatomy examined by cryomicrotome section. Influence of age, vertebral level, and disease. Reg Anesth 1996; 21: 395-406.

15. Pan PH, Bogard TD, Owen MD. Incidence and characteristics of failures in obstetric neuraxial analgesia and anesthesia: a retrospective analysis of 19,259 deliveries. Int J Obstet Anesth 2004; 13: 227-33.

16. Mhyre JM, Greenfield ML, Tsen LC, Polley LS. A systematic review of randomized controlled trials that evaluate strategies to avoid epidural vein cannulation during obstetric epidural catheter placement. Anesth Analg 2009; 108: 1232-42.

17. Evron S, Gladkov V, Sessler DI, Khazin V, Sadan O, Boaz M, et al. Predistension of the epidural space before catheter insertion reduces the incidence of intravascular epidural catheter insertion. Anesth Analg 2007; 105: 460-4.

18. Banwell BR, Morley-Forster P, Krause R. Decreased incidence of complications in parturients with the arrow (FlexTip Plus) epidural catheter. Can J Anaesth 1998; 45: 370-2.

19. Bell DN, Leslie K. Detection of intravascular epidural catheter placement: a review. Anaesth Intensive Care 2007; 35: 335-41.

20. Furman MB, Giovanniello MT, O'Brien EM. Incidence of intravascular penetration in transforaminal cervical epidural steroid injections. Spine (Phila Pa 1976) 2003; 28: 21-5.

21. Smuck M, Fuller BJ, Yoder B, Huerta J. Incidence of simultaneous epidural and vascular injection during lumbosacral transforaminal epidural injections. Spine J 2007; 7: 79-82.

22. Kim DW, Shim JC. Incidence of intravascular penetration during 
transforaminal lumbosacral epidural steroid injection. Korean J Pain 2007; 20: 26-30.

23. Kim do W, Han KR, Kim C, Chae YJ. Intravascular flow patterns in transforaminal epidural injections: a comparative study of the cervical and lumbar vertebral segments. Anesth Analg 2009; 109: 233-9.
24. Choi IS, Berenstein A. Surgical neuroangiography of the spine and spinal cord. Radiol Clin North Am 1988; 26: 1131-41.

25. Lee MH, Yang KS, Kim YH, Jung HD, Lim SJ, Moon DE. Accuracy of live fluoroscopy to detect intravascular injection during lumbar transforaminal epidural injections. Korean J Pain 2010; 23: 18-23. 